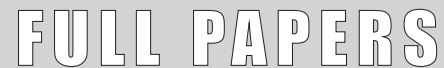

\section{Cervico-vaginal monitoring in pregnancy in Italy}

\author{
Antonietta Cavallaro', Annalisa Trevisan', Carlo Mengoli',2 \\ ' Servizio di Microbiologia e Virologia dell'Ospedale di Padova, Italy \\ ${ }^{2}$ Dipartimento di Istologia, Microbiologia e Biotecnologie Mediche, Università di Padova, Italy
}

Key Words: RTIs, pregnancy, Chlamydia trachomatis, Trichomonas vaginalis, Listeria monocytogenes, Streptococcus agalactiae

II monitoraggio microbiologico cervico-vaginale in gravidanza su popolazione italiana e straniera

\section{SUMMARY}

\section{Background}

Reproductive tract infections (RTIs) in pregnancy are an important cause of fetal and neonatal morbidity, sometimes with serious complications. Chlamydia trachomatis causes ectopic pregnancy, conjunctivitis, pneumonia and RTIs in newborn babies. Listeria monocytogenes and Streptococcus agalactiae can give neonatal sepsis and meningitis, and Trichomonas vaginalis causes premature birth.

\section{Objectives}

To update local epidemiology of RTIs in pregnancy in order to to implement efficacious screening and prevention programs.

\section{Methods}

Between January- December 2006, at the Padua's Hospital Microbiology and Virology Service, we investigated 2000 asimptomatic pregnant women, 18-45 years old, Italian and foreigners. We collected vaginal and cervical swabs for the microscopy, the culture for Neisseria gonorrhoeae, Trichomonas vaginalis, Mycoplasma spp., Streptococcus agalactiae, Listeria monocytogenes, and other bacteria and yeasts, and for the molecular assay for Chlamydia trachomatis.

\section{Results}

The prevalence of Chlamydia trachomatis and Trichomonas vaginalis in our population were $2.06 \%$ and $0.20 \%$, respectively. Neisseria gonorrhoeae was not detected. Sexually transmitted infections (STIs) were more frequent among foreigners, and in women aged 18-30. The prevalence of Streptococcus agalactiae was $8.69 \%$ for Italian, and $7.54 \%$ for foreigners. We didn't find any case of maternal colonization by Listeria monocytogenes.

\section{Conclusions}

From the obtained results ideas have emerged to arrange a qualitative and quantitative optimization of the diagnosis of RTIs, implementing diagnostic paths based on the different typologies of patients and on the local epidemiology. The cultural research of Listeria monocytogenes takes a long time, perhaps a molecular one, because of fastness and sensitivity, could be more useful.

\section{Received August 8, 2008}

Accepted February 27, 2009

\section{INTRODUZIONE}

In gravidanza, le infezioni del tratto riproduttivo (RTIs) o la colonizzazione vaginale da parte di microrganismi quali Streptococcus agalactiae o Listeria monocytogenes, possono essere causa di morbilità fetale e neonatale con sequele molto gravi. La sepsi o la meningite causate da Listeria monocytogenes o da Streptococcus agalactiae, ad es., sono correlate a significativi tassi di mortalità neonatale. Inoltre, le RTIs acquisite durante la gestazione hanno spesso un'influenza negativa sull'esito della gravidanza stessa, quale maggiore rischio di aborto, di parto prematuro, di amnioni- te, gravidanza ectopica $(1,12,13,20)$. Il problema è presente in misura maggiore nei paesi con basso tenore socio-economico e ridotta assistenza sanitaria, nei quali risulta più difficile implementare programmi di screening e prevenzione ante-parto. Inerentemente questi paesi, infatti, la letteratura presenta dati di prevalenza significativamente elevati per infezioni da agenti etiologici di STIs $(4,6)$ sebbene l'entità degli attuali flussi migratori contribuisca ad estendere il problema anche ai paesi sede di immigrazione. La sorveglianza microbiologica rappresenta un punto fondamentale nel controllo e nella prevenzione delle infezioni feta-

\section{Corresponding author: Antonietta Cavallaro}

A.O. di Padova - Servizio di Microbiologia e Virologia

Via Giustiniani, 2 - 35I00 Padova, Italy - Tel. 04982 I3049 - Fax 82I3054

E-mail: antonietta.cavallaro@sanita.padova.it 
li e perinatali, i cui responsabili possono essere agenti eziologici di STIs ma anche microrganismi colonizzanti quali Streptococcus agalactiae, Mycoplasma spp., e Listeria monocytogenes. Al fine di ottimizzare programmi di screening e prevenzione sono essenziali dati di epidemiologia su base locale che evidenzino i principali agenti di RTIs in relazione a fattori di rischio (età, provenienza o etnicità,...) delle gestanti. Il nostro studio offre un aggiornamento a livello territoriale dell'epidemiologia dei microrganismi isolati da tampone vaginale, vagino/rettale, e da tampone cervicale di gravide asintomatiche di nazionalità italiana e straniera, da cui possono emergere spunti di riflessione per percorsi diagnostici diversificati per tipologia di paziente.

\section{MATERIALI E METODI}

\section{Popolazione analizzata}

Lo studio era condotto su una popolazione costituita da 2000 gravide, asintomatiche, afferenti al Servizio di Microbiologia e Virologia dell'Ospedale di Padova per indagini microbiologiche di screening pre-natale nel periodo gennaio - dicembre 2006. Le pazienti avevano età compresa tra 18 e 45 anni, e nazionalità Italiana o provenienza extracomunitaria o comunitaria (in prevalenza dall'Europa dell'Est).

\section{Prelievo}

Venivano eseguiti tre tamponi vaginali, da fornice posteriore, uno per la microscopia, e due per le indagini colturali (uno per Listeria monocytogenes, e uno per miceti, batteri e Trichomonas vaginalis), e un tampone cervicale per ricerca di Neisseria gonorrhoeae. Per Streptococcus agalactiae si effettuava un tampone vagino/rettale. Chlamydia trachomatis e Mycoplasma spp. erano ricercati su specifica richiesta del medico curante, effettuando un tampone cervicale per ciascuna delle due indagini.

\section{Microscopia}

Per ogni paziente si allestiva un preparato per la colorazione al Gram, che veniva esaminato al microscopio, a 100X (10). La diagnosi di vaginosi era effettuata sulla base della microscopia del preparato da tampone vaginale colorato al Gram, secondo i criteri di Nugent (19), la presenza delle tipiche "clue cells"con adesi numerosi microrganismi cocco-bacillari Gram variabili, e l'assenza di leucociti.

\section{Indagini colturali}

Il tampone vaginale era seminato su Columbia agar + sangue di montone 5\%, "COS" (bioMérieux), su agar cioccolato + PolyViteX, "PVX" (bioMérieux) per la ricerca di Haemophilus spp., e su agar
"Can2" (bioMérieux) per la ricerca di miceti. Il tampone vagino/rettale per Streptococcus agalactiae era seminato su CNA-agar sangue di montone $5 \%$, "CNA" (bioMérieux). Le piastre erano incubate per 48 ore a $37^{\circ} \mathrm{C}$ in diverse condizioni d'atmosfera: il "COS" (bioMérieux) e il CNA-agar sangue di montone 5\%, "CNA" (bioMérieux) in anaerobiosi, l'agar cioccolato "PolyViteX" (bioMérieux) in atmosfera arricchita in $\mathrm{CO}_{2}$ al 5\%, il "Can2 agar" (bioMérieux) in aerobiosi. Quest'ultimo terreno, dopo incubazione, consentiva una rapida identificazione presuntiva delle specie di Candida sulla base della colorazione assunta dalle colonie (ad es. azzurro scuro per Candida albicans).

L'identificazione delle Candida non albicans era invece effettuata con la card "YST" Vitek (bioMérieux).

\section{Neisseria gonorrhoeae}

Per la ricerca di Neisseria gonorrhoeae si procedeva alla semina del tampone cervicale su agar cioccolato + PolyViteX + VCA, "VCA3" (bioMérieux). Tale terreno era incubato a $37^{\circ} \mathrm{C}$ in atmosfera arricchita in $\mathrm{CO}_{2}$ al $5 \%$ per 48 ore.

Dopo 24 e 48 ore d'incubazione, le piastre di "VCA3" erano esaminate per la presenza di colonie dall'aspetto traslucido, sopraelevate, lucenti, trasparenti od opache. Dalle colonie con caratteristiche tipiche di N.gonorrhoeae si procedeva all'esecuzione della prova della catalasi e dell'ossidasi, e all'allestimento di un vetrino al Gram. Se il test della catalasi e quello dell'ossidasi risultavano positivi, e se il vetrino evidenziava cocchi Gram negativi, si effettuava l'arricchimento dell'isolato in agar cioccolato "PolyViteX", "PVX" (bioMérieux) con incubazione per 24 ore a $37^{\circ} \mathrm{C}$ in atmosfera arricchita di $\mathrm{CO}_{2}$ al $5 \%$, sul quale si eseguiva poi l'identificazione biochimica con il sistema "Api NH” (bioMérieux).

\section{Trichomonas vaginalis}

Per la ricerca di Trichomonas vaginalis (16) il tampone vaginale era seminato in Trichomonas Selective Broth (Labobasi) che veniva incubato a $37^{\circ} \mathrm{C}$ per 5 giorni. Ogni 24 ore, per 5 giorni consecutivi, si procedeva all'osservazione microscopica a 40X di un'aliquota del brodo inoculato.

\section{Streptococcus agalactiae}

Il tampone vagino/rettale per la ricerca di Streptococcus agalactiae era seminato su CNAagar sangue di montone 5\%, "CNA" (bioMérieux). Le piastre erano incubate a $37^{\circ} \mathrm{C}$, in atmosfera anaerobia. Dopo 24 ore, le colonie che presentavano un alone di beta emolisi, sospette per Streptococcus agalactiae, erano identificate con l'antisiero AB Phadebact ${ }^{\circledR}$ Strep B (Boule 
Diagnostics). L'antibiogramma per Streptococcus agalactiae veniva eseguito di routine su tutti gli isolati, ed era allestito con il metodo Vitek 2 (bioMérieux).

\section{Mycoplasma spp.:}

Ureaplasma urealyticum e Mycoplasma hominis erano ricercati con metodo colturale "Mycoplasma IST 2" (bioMérieux) che consente di effettuare contemporaneamente la coltura, l'identificazione, e le prove di sensibilità agli antimicrobici. La lettura della galleria era effettuata a 24 ore e a 48 ore, dopo incubazione a $37^{\circ} \mathrm{C}$, secondo le indicazioni fornite dalla casa produttrice (bioMérieux).

\section{Listeria monocytogenes}

La ricerca di Listeria monocytogenes da tampone vaginale era effettuata con un metodo colturale basato sul protocollo del Netherlands Government Food Inspection Service, NGFIS, (22) impiegato anche dal CDC per isolare L. monocytogenes da campioni clinici da siti non sterili. Il metodo dell'NGFIS ha una sensibilità pari al 75\% (5). Esso prevedeva l'arricchimento del campione in un brodo selettivo, Listeria PALCAM-egg yolk (Labobasi), incubato a $30^{\circ} \mathrm{C}$ per un totale di 48 ore. Dopo 24 e 48 ore rispettivamente, si effettuava la semina di un'aliquota dell'arricchimento in terreno solido selettivo, Listeria PALCAM agar (Labobasi) con incubazione per 24 ore a $30^{\circ} \mathrm{C}$, in microaerofilia. Le piastre erano esaminate dopo 24 ore per la presenza di colonie dall' aspetto compatibile con Listeria (colorazione grigio-verde, con un alone nero, e un diametro di circa $2 \mathrm{~mm}$ ) sulle quali erano allestite la prova della catalasi, un vetrino colorato al Gram, ed eventualmente un'identificazione con galleria "Api Coryne" (bioMérieux) la cui lettura era effettuata dopo un'incubazione di 24 ore a $37^{\circ} \mathrm{C}$, in aerobiosi.

\section{Indagini di biologia molecolare}

La ricerca di Chlamydia trachomatis da tampone cervicale era effettuata con la tecnica di biologia molecolare "BD ProbeTec ET2" (BecktonDickinson) (3). Tale metodo, per la sua elevata sensibilità, è da anni in uso presso il Servizio di Microbiologia e Virologia dell'Ospedale di Padova in sostituzione del metodo in immunofluorescenza (9). Questa tecnica è qualitativa e consiste in un'amplificazione del DNA secondo il principio SDA (Strand Displacement Amplification) (17). La rilevazione dell' amplificato avviene durante la fase stessa di amplificazione, tramite una specifica sonda fluorescente. A seconda della popolazione studiata, questa tecnica ha una specificità del 95.6\%-97.6\% e una sensibilità dell' $88.9 \%-98.4 \%$ per campioni cervicali (3). Il prelievo veniva effet- tuato con un apposito tampone incluso nella confezione dei reattivi forniti.

\section{Analisi statistica}

L'analisi statistica dei dati era effettuata con il test del $\chi^{2}$ (ad un grado di libertà) o il test esatto di Fisher (solo per la valutazione dei dati su Trichomonas vaginalis, in cui uno dei conteggi relativi alle variabili binomiali a confronto era pari a zero).

\section{RISULTATI}

Lo studio esaminava una popolazione di 2000 donne in gravidanza, asintomatiche, di nazionalità italiana $\mathrm{e}$ straniera, e di età compresa tra 18 e 45 anni.

I dati relativi al totale della popolazione esaminata sono riportati nella Tabella 1.

Tabella I. Esito delle indagini microbiologiche sul totale della popolazione

\begin{tabular}{|c|c|}
\hline POSITIVO & $712(35,60 \%)$ \\
\hline NEGATIVO & $1288(64,40 \%)$ \\
\hline \multicolumn{2}{|l|}{ Prevalenza patogeni STIs } \\
\hline Chlamydia trachomatis $* *$ & $12(2,06 \%)$ \\
\hline Trichomonas vaginalis & $4(0,20 \%)$ \\
\hline \multicolumn{2}{|l|}{ Neisseria gonorrhoeae } \\
\hline \multicolumn{2}{|l|}{ Prevalenza microrganismi non STIs } \\
\hline Ureaplasma urealyticum ** & $160(27,49 \%)$ \\
\hline Candida spp. & $244(12,20 \%)$ \\
\hline Streptococcus agalactiae & $168(8,40 \%)$ \\
\hline Gardnerella vaginalis & $74(3,70 \%)$ \\
\hline Mycoplasma hominis ** & $8(1,37 \%)$ \\
\hline \multicolumn{2}{|l|}{ Listeria monocytogenes } \\
\hline Altri microrganismi non STls & $42(2,1 \%)$ \\
\hline \multicolumn{2}{|l|}{ ** tot. soggetti esaminati per } \\
\hline C. trachomatis, U. urealyticum, M. hominis & $582(29,10 \%)$ \\
\hline
\end{tabular}

(Le ricerche di Chlamydia trachomatis, Mycoplasma hominis, ed Ureaplasma urealyticum, erano effettuate su una sottopopolazione di 582 gravide).

La percentuale d'indagini microbiologiche positive era pari a $35.60 \%$ (712 soggetti), il $64.40 \%$ risultava invece negativo (1288 donne). Sul totale della popolazione esaminata, si riscontravano 16 casi d'infezione da patogeni sessualmente trasmessi. In ordine di prevalenza, Chlamydia trachomatis $(2.06 \%)$ era il principale agente eziologico di STIs rilevato, seguito da Trichomonas vaginalis con uno $0.20 \%$. Non si registrava invece alcuna positività per Neisseria gonorrhoeae.

Tra i microrganismi non STIs prevalevano Ureaplasma urealyticum (27.49\%), Candida spp. (12.20\%) e Streptococcus agalactiae (8.40\%). Non si riscontrava invece alcuna positività per Listeria monocytogenes. Per quanto riguarda l'antibiogramma per Streptococcus agalactiae, si rile- 
vava un $15 \%$ di resistenza all'eritromicina e un $15 \%$ alla clindamicina; tutti gli isolati erano sensibili alla Penicillina.

In Tabella 2 vengono riepilogati i dati ottenuti sulla base della ripartizione della popolazione per nazionalità. Il $74.80 \%$ delle gravide (1496 soggetti) era di nazionalità italiana, mentre il $25.20 \%$ (504 donne) era di nazionalità straniera. Per $C$. trachomatis, $M$. hominis, ed U.urealyticum, erano state esaminate rispettivamente 384 donne italiane e 198 straniere.
La percentuale d'indagini ad esito positivo era più elevata tra le gravide straniere $(50.79 \%$ in confronto al $30.48 \%$ delle italiane, $\mathrm{p}=2.77 \mathrm{E}-16)$. Si riscontravano rilevanti differenze nelle prevalenze dei patogeni STIs in rapporto alla nazionalità. Per Chlamydia trachomatis, la positività era nettamente superiore tra le straniere $(5.05 \%$ rispetto allo $0.52 \%$ delle italiane, $\mathrm{p}=0.000852$ ) e la discrepanza era ancora più significativa se si considera che il numero di donne straniere esaminate per questo microrganismo era circa la metà di quello

Tabella 2. Nazionalità ed esito delle indagini microbiologiche

\begin{tabular}{|c|c|c|c|}
\hline & \multicolumn{2}{|c|}{ Nazionalità } & \multirow[t]{2}{*}{$\mathbf{P}$} \\
\hline & Italiana & Straniera & \\
\hline POSITIVO & $456(30,48 \%)$ & $256(50,79 \%)$ & $2,77 \mathrm{E}-16$ \\
\hline NEGATIVO & $1040(69,52 \%)$ & $248(49,21 \%)$ & \\
\hline TOTALE & $1496(74,80 \%)$ & $504(25,20 \%)$ & \\
\hline \multicolumn{4}{|l|}{ Prevalenza dei patogeni STIs } \\
\hline Chlamydia trachomatis $* *$ & $2(0,52 \%)$ & $10(5,05 \%)$ & 0,000852 \\
\hline Trichomonas vaginalis & & $4(0,79 \%)$ & 0,0040 \\
\hline \multicolumn{4}{|l|}{ Neisseria gonorrhoeae } \\
\hline \multicolumn{4}{|c|}{ Prevalenza dei microrganismi non STIs } \\
\hline Ureaplasma urealyticum ** & $104(27,08 \%)$ & $56(28,28 \%)$ & NS \\
\hline Candida spp. & $144(9,62 \%)$ & $100(19,84 \%)$ & $2,2 \mathrm{IE}-09$ \\
\hline Streptococcus agalactiae & $130(8,69 \%)$ & $38(7,54 \%)$ & NS \\
\hline Gardnerella vaginalis & $42(2,8 I \%)$ & $32(6,35 \%)$ & 0,000454 \\
\hline Mycoplasma hominis ** & $2(0,52 \%)$ & $6(3,03 \%)$ & 0,036823 \\
\hline \multicolumn{4}{|l|}{ Listeria monocytogenes } \\
\hline Altri microrganismi non STls & $32(2,14 \%)$ & $10(1,98 \%)$ & NS \\
\hline ** tot. soggetti esaminati per & & & \\
\hline C. trachomatis, U. urealyticum, M.hominis & $384(25,67 \%)$ & $198(39,28 \%)$ & \\
\hline
\end{tabular}

Tabella 3. Età ed esito delle indagini microbiologiche

\begin{tabular}{|c|c|c|c|}
\hline & \multicolumn{2}{|c|}{ Età } & \multirow[t]{2}{*}{$\mathbf{P}$} \\
\hline & 18-30 anni & 3 I-45 anni & \\
\hline POSITIVO & $268(50 \%)$ & $444(30,33 \%)$ & $6,2 \mathrm{E}-16$ \\
\hline NEGATIVO & $268(50 \%)$ & $1020(69,67 \%)$ & \\
\hline TOTALE & $536(26,80 \%)$ & $1464(73,20 \%)$ & \\
\hline \multicolumn{4}{|l|}{ Prevalenza dei patogeni STIs } \\
\hline Chlamydia trachomatis $* *$ & $10(11,11 \%)$ & $2(0,99 \%) ?$ & 0,000222 \\
\hline Trichomonas vaginalis & $4(0,75 \%)$ & & 0,0052 \\
\hline \multicolumn{4}{|l|}{ Neisseria gonorrhoeae } \\
\hline \multicolumn{4}{|c|}{ Prevalenza dei microrganismi non STIs } \\
\hline Ureaplasma urealyticum $* *$ & $58(64,44 \%)$ & $102(50,75 \%)$ & 0,041013 \\
\hline Candida spp. & $88(16,42 \%)$ & $156(10,65 \%)$ & 0,000649 \\
\hline Streptococcus agalactiae & $50(9,33 \%)$ & II8 (8,06\%) & NS \\
\hline Gardnerella vaginalis & $42(7,83 \%)$ & $32(2,18 \%)$ & $6,82 \mathrm{E}-09$ \\
\hline Mycoplasma hominis ** & $6(6,66 \%)$ & $2(0,99 \%)$ & 0,018924 \\
\hline \multicolumn{4}{|l|}{ Listeria monocytogenes } \\
\hline Altri microrganismi non STls & $10(1,86 \%)$ & $32(2,18 \%)$ & NS \\
\hline \multicolumn{4}{|l|}{ ** totale soggetti esaminati per } \\
\hline C. trachomatis, U. urealyticum, M.hominis & $90(16,79 \%)$ & $20 \mathrm{I}(13,73 \%)$ & \\
\hline
\end{tabular}


delle autoctone. Per Trichomonas vaginalis, invece, si registrava una prevalenza pari a $0.79 \%$ tra le gravide non autoctone $(\mathrm{p}=0.0040)$, mentre non si avevano casi di tricomoniasi tra le gravide italiane. La prevalenza di Chlamydia da noi rilevata nelle donne non autoctone è in accordo con i dati di altri autori inerenti paesi africani : $5.9 \%$ in uno studio condotto da Mayaud et al. in Tanzania, nel 1998 (18); 6.2\% in uno studio di Blankhart et al. del 1996, nella Repubblica Centrale Africana (6). Per l'Europa dell'Est, invece, la letteratura (21) riporta percentuali di positività inferiori (2.5\% in Polonia, nel 2005, secondo Pawlowska et al).

Tra i microrganismi non STIs, le prevalenze di Candida spp., Gardnerella vaginalis, e Mycoplasma hominis erano significativamente più elevate tra le gravide non autoctone $(19.84 \%, 6.35 \%$,e $3.03 \%$ in confronto a $9.26 \%, 2.81 \%$, e $0.52 \%$ nelle italiane; $\mathrm{p}=2.21 \mathrm{E}-09, \mathrm{p}=0.000454$, e $\mathrm{p}=0.036823$ rispettivamente). Relativamente alla prevalenza di Streptococcus agalactiae riscontrata nella popolazione autoctona esaminata ( $8.69 \%$ ), i nostri dati sono in accordo con quanto riportato da Bagnani et al, in uno studio condotto nel Nord-Est d'Italia, in cui il tasso di positività registrato per Streptococcus agalactiae nelle gestanti era pari a $8.18 \%$ (2). Citernesi et al, invece, in uno studio su gravide italiane condotto nel 1996, riportavano una prevalenza pari al $6.6 \%$ (11). Per quanto riguarda le gravide straniere, la percentuale di Streptococcus agalactiae da noi rilevata appare inferiore a quanto riportato da altri autori. Ad es., in uno studio su 1678 gravide condotto in Polonia nel 2003, Kowalska et al. registravano uno $19.7 \%$ di positività (15).

La Tabella 3 riassume l'esito delle indagini sulla base della ripartizione della popolazione in fasce d'età. L'età media delle gravide autoctone era 34 anni, mentre per le donne extracomunitarie o comunitarie era 29 anni. Le ricerche di $C$. trachomatis, $M$. hominis, ed U.urealyticum, erano state effettuate rispettivamente su un totale di 90 donne tra i 18 e 30 anni, e di 201 tra 31 e 45 anni.

Le indagini microbiologiche positive prevalevano tra le donne di età $18-30$ anni $(50 \%$ in confronto a $30.33 \%$ della fascia d'età superiore, $p=6.2 \mathrm{E}-16$ ). Le infezioni da patogeni STIs erano significativamente più elevate tra le gravide più giovani $(\mathrm{p}=0.000222$ per Chlamydia, $\mathrm{p}=0.0052$ per Trichomonas). Tutti i casi di tricomoniasi erano registrati solo tra i soggetti di età 18-30 anni. Inoltre, su un totale di 12 casi di Chlamydia trachomatis, 10 erano riscontrati nella fascia d'età più bassa. Il dato di prevalenza per Chlamydia tra le gravide più giovani $(11.11 \%$ in confronto a $0.99 \%$ delle donne d'età più avanzata) è particolarmente significativo anche alla luce del numero totale di donne investigate per questo patogeno (la metà di quello delle più anziane).
Si rilevava una maggior positività tra le gravide di età 18-30 anni anche per Candida spp., Gardnerella vaginalis, Ureaplasma urealyticum, e Mycoplasma hominis.

\section{DISCUSSIONE}

Il nostro studio fornisce un aggiornamento su base locale dell'epidemiologia di microrganismi isolati da tampone vaginale, vaginorettale, e da tampone cervicale in gravide asintomatiche, e può offrire spunti per implementare percorsi diagnostici diversificati per il monitoraggio microbiologico in gravidanza, basati sull'epidemiologia locale e volti ad un approccio personalizzato al paziente.

Sulla base dei dati di prevalenza riscontrati, riteniamo un caposaldo irrinunciabile della sorveglianza microbiologica nelle gravide la ricerca di Chlamydia trachomatis e Streptococcus agalactiae, a prescindere da età e nazionalità delle gestanti. Chlamydia trachomatis dovrebbe essere ricercata al primo screening in gravidanza, o meglio ancora in fase preconcezionale, per meglio contribuire alla riduzione di casi di gravidanza ectopica, e di aborto ad essa legati. Per Streptococcus agalactiae, invece, la ricerca dovrebbe essere condotta verso la $35^{\mathrm{a}}-37^{\mathrm{a}}$ settimana di gestazione (8) in quanto un trattamento lontano dal parto, seppure adeguato, non consente l'eradicazione della colonizzazione (14) mentre la somministrazione di antibiotico per via endovenosa al momento del travaglio, nelle gravide positive, risulta fondamentale nella prevenzione di polmonite, sepsi, o meningite neonatali (8). Sebbene le linee guida del CDC non ritengano necessario l'antibiogramma, la percentuale di resistenza riscontrata ai macrolidi, valida alternativa nei pazienti allergici alla penicillina, ci induce a considerarlo una preziosa informazione per il clinico, soprattutto in mancanza di dati anamnestici al riguardo, quantomeno per la popolazione locale. Il nostro lavoro ha cercato di indagare sulla prevalenza di Listeria monocytogenes in gravidanza, sulla quale pochi sono gli studi disponibili $(7,13)$. Sebbene nella popolazione esaminata non siano stati rilevati casi di listeriosi materna o di colonizzazione vaginale da Listeria monocytogenes, riteniamo comunque importante la ricerca di questo microrganismo. Poiché il protocollo colturale può rivelarsi indaginoso, sarebbe auspicabile lo sviluppo di un metodo di biologia molecolare (real time PCR) che consenta, grazie ad elevate sensibilità e specificità, un'agevole rilevazione di Listeria monocytogenes da un campione polimicrobico quale il tampone vaginale, anche se presente in bassa carica. Di particolare importanza e utilità, sarebbe inoltre la capacità di una real time PCR di fornire un responso nell'arco di poche ore, 
ovvero in tempo reale, così da poter eventualmente istituire un'adeguata terapia tempestivamente. Lo sviluppo di un'indagine per Listeria del tipo NAAT (nucleic acid amplification test) da allestire in pool, e il suo inserimento nel programma di sorveglianza microbiologica, all'ultima settimana di gravidanza, potrebbero rappresentare un buon compromesso tra la necessità di prevenire gravi infezioni neonatali, causate da colonizzazione vaginale asintomatica materna, e la necessità di ottimizzazione quantitativa nella diagnostica prenatale. Nelle gestanti straniere, è inoltre opportuno porre particolare attenzione alla ricerca di Trichomonas vaginalis, soprattutto nella fascia d'età 18-30 anni, impiegando un metodo ad elevata sensibilità quale l'esame colturale o tecniche di biologia molecolare. Inoltre, per le donne in gravidanza non autoctone, ai fini della riduzione dei casi di RTIs e delle loro complicanze, sarebbe rilevante la presenza nel territorio di presidi con attività di counseling che, pur nel rispetto delle variabili socio-economiche dei paesi di origine dei soggetti, inducano un maggior avvicinamento ai programmi sanitari di screening e prevenzione.

\section{BIBLIOGRAFIA}

1. Abele-Horn M, Peters J, Genzel-Boroviczeny O, et al. Vaginal Ureaplasma urealyticum colonization: influence on pregnancy outcome and neonatal morbidity. Infect 1997; 25(5): 286-91

2. Bagnani A, Battisti E, Battistini A, et al. Prevalence of group B beta-hemolytic Streptococcus colonization in a sample of 23,312 pregnant women and newborn infants. Pediatr Med Chir 1995; 17(4): 295-7

3. BD ProbeTec TM ET Chlamydia trachomatis Amplified DNA Assay. Becton Dickinson and Company, Loveton Circle Sparks, MD 2004; 89

4. Begum A, Nilufar S, Akther K, et al. Prevalence of selected reproductive tract infections among pregnant women attending an urban maternal and childcare unit in Dhaka, Bangladesh. J Health Popul Nutr 2003; 21(2): 112-6

5. Bille J, Rocourt J, Swaminathan B. Listeria and Erysipelothrix. In: Murray P, Baron EJ, Jorgensen JH, Pfaller MA, Yolken RH Eds. Manual of Clinical Microbiolgy, Eight Edition. ASM Press, Washington DC, 2003: 463

6. Blankhart D, Muller O, Gresenguet G, et al. Sexually transmitted infections in young pregnant women in Bangui, Central African Republic. Int J STD AIDS 1999; Sep. 10(9): 609-14

7. Campello C, Crevatin E, Elia A, et al. Human listeriosis in the Trieste area. Boll Ist Sieroter Milan 1981; Jul 60(3): 198-205

8. CDC, Center for Disease Control and Prevention. Prevention of perinatal group B streptococcal disease.
Revised guidelines from CDC. CDC Atlanta 2002; MMWR 51(RR-11)

9. CDC, National Center for HIV, STD and TB Prevention, Division of Sexually Transmitted Diseases. 2002 laboratory guidelines screening tests to detect Chlamydia trachomatis and Neisseria gonorrhoeae infections. In: STD prevention. CDC Atlanta 2002; 2-4

10. Chapin CK, Lauderdale TL. Reagents, stains, and media: bacteriology. In: Murray $\mathrm{P}$, Baron EJ, Jorgensen JH, Pfaller MA, Yolken RH Eds. Manual of Clinical Microbiolgy, Eight Edition. ASM Press, Washington DC, 2003: 363

11. Citernesi A, Formica G, Caruso S, et al. Vaginal colonization of Streptococcus B in pregnancy. Min Ginecol 1996; 48(6): 227-33

12. Cotch MF, Pastorek JG, Krieger J, et al. Trichomonas vaginalis associated with low birth weight and preterm delivery. The vaginal infections and prematurity study group. Sex Transm Dis 1997; 24: 353-60

13. Frederiksen B, Samuelsson S. Feto-maternal listeriosis in Denmark 1981-1988. J Infect 1992; 24(3): 277 87.

14. Gardner SE, Yow MD, Leeds LJ, et al. Failure of penicillin to eradicate group B streptococcal colonization in the pregnant woman: a couple study. Am J Obstet Gynecol 1979; 135: 1062-5

15. Kowalska B, Niemiec KT, Drejewicz H, et al. Prevalence of group B streptococcal colonization in pregnant women and their newborns based on the results of examination of patients in the Obstetric and Gynecology Department of the National Research Institute of Mother and Child - a pilot study. Ginekol Pol 2003;74(10): 1223-7

16. Leber AL, Novak SM. Intestinal and urogenital amebae, flagellates, and ciliates. In: Murray P, Baron EJ, Jorgensen JH, Pfaller MA, Yolken RH Eds. Manual of Clinical Microbiolgy, Eight Edition. ASM Press, Washington DC, 2003: 2002

17. Littlea MC. Strand Displacement Amplification and homogeneous real-time detection incorporated in a second generation DNA probe system BD ProbeTec ET. Clin Chem 1999; 45(6): 777-84

18. Mayaud P, Uledi E, Cornelissen J, et al. African Medical Research Foundation (AMREF), Mwanza, Tanzania. Risk scores to detect cervical infections in urban antenatal clinic attenders in Mwanza, Tanzania. Sex Transm Infect. 1998; 74 (1):S139-46

19. Nugent RP, Krohn MA, Hillier SL. Reliability of diagnosing bacterial vaginosis is improved by a standardized method of Gram stain interpretation. J Clin Microbiol 1991; 29: 297-301.

20. Pass MA, Gray BM, Dillon HC. Puerperal and perinatal infections with group B Streptococci. Am J Obstet Gynecol 1982; 143: 147-52

21. Pawlowska A, Niemiec KT, Filipp E, et al. Chlamydia trachomatis infection in pregnant women hospitalised in the Institute of Mother and Child in Warsaw, Poland. Med Wieku Rozwoj 2005; 9(1): 21-6

22. Van Netten P, Perales I, van de Moosdijk A, et al. Liquid and solid differential media for the detection and enumeration of Listeria monocytogenes and other Listeria spp. Int J Food Microbiol 1989; 8: 299-316 